\title{
LITERATUR
}

\section{Transatlantische Beziehungen nach 9/11 und dem Krieg im Irak}

\author{
Uli Brückner**
}

Bis zum Ende des Kalten Krieges war klar, was unter, Westen' zu verstehen war, denn es gab einen Gegenentwurf und in globaler Perspektive auch noch die sogenannte Dritte Welt. Diese Grundüberzeugung zieht sich durch transatlantische Studien jener Zeit. In den 1990er Jahren schwindet diese Gewissheit und es beginnt die Suche nach den ideellen Grundlagen für die Zukunft des Verhältnisses. Anknüpfungspunkte dieser Debatte sind zum Beispiel die Entwicklung der Gemeinsamen Außen- und Sicherheitspolitik (GASP) im Verhältnis zur NATO, das Engagement der USA auf dem Balkan oder der Beginn des Globalisierungsdiskurses. Wissenschaftliche und politische Positionen in Europa bewegen sich zwischen dem Bekenntnis zur Fortsetzung einer, alternativlosen' atlantischen Partnerschaft und einer politischen Weiterentwicklung eines integrierten Europas in Richtung eines selbstständigen globalen Akteurs, sowie der Frage, ob dies ein Widerspruch sein muss. Werner Weidenfeld forderte 1996 eine Neubestimmung der transatlantischen Agenda, die ,wenn sie erfolgreich sein will, beiden Partnern nicht nur eine gemeinsame Agenda zweckrationalen Handelns liefern, sondern ihnen auch eine Neubestimmung ihrer Identitäten ermöglichen (muss), die den Partner auf der anderen Seite miteinbezieht" ${ }^{1}$ So könnte man das Programm umschreiben, an dem Politik und Wissenschaft in Europa seither arbeiten, auch wenn sich seit Mitte der 1990er Jahre nicht nur Personen und Kontext verändert haben.

Thomas Jäger, Alexander Höse und Kai Oppermann (Hrsg.): Transatlantische Beziehungen. Sicherheit - Wirtschaft - Öffentlichkeit, VS Verlag für Sozialwissenschaften: Wiesbaden 2005, ISBN 3-531-14579-7; 540 Seiten, $41 €$.

Nikos Kotzias und Petros Liacouras (Hrsg.): EUUS Relations. Repairing the Transatlantic Rift, Palgrave Macmillan: Houndmills Basingstoke 2006, ISBN 10: 1-4039-3521-1; 263 Seiten, $19.99 £$.

Roland Dannreuther und John Peterson (Hrsg.): Security Strategy and Transatlantic Relations, Routledge: London/New York 2006, ISBN 10: 0-415-40189-5; 262 Seiten, $19.99 £$.

Lazaros Miliopoulos: Atlantische Zivilisation und transatlantisches Verhältnis. Politische Ideen und Wirklichkeit, VS Verlag für Sozialwissenschaften: Wiesbaden 2007, ISBN 978-3-53115292-9; 510 Seiten, 64,90 €.

Vordergründig nehmen alle jüngeren Veröffentlichungen zu transatlantischen Beziehungen 9/11 und den Irakkrieg als zentrale Bezugspunkte. Hinter der Analyse und Bewertung dieser Ereignisse steht jedoch die Frage nach der gemeinsamen Agenda und ihrer Grundlagen.

Die hier besprochenen Bücher sind nicht mit der Absicht eines repräsentativen Überblicks über den Stand der Forschung ausgewählt. Dafür ist schon die Auswahl zu klein. Auch stammen alle Veröffentlichungen aus Europa, wenngleich zahlreiche amerikanische Auto-

* Dr. Uli Brückner, Jean Monnet Chair, Stanford University.

1 Werner Weidenfeld: Kulturbruch mit Amerika? Das Ende transatlantischer Selbstverständlichkeit, Gütersloh 1996, S. 11. 
ren vertreten sind. Vor allem handelt es sich um sehr unterschiedliche Textgenres: eine Dissertation, ein Konferenzband, das Ergebnis eines Forschungsprojekts und eine Aufsatzsammlung.

\section{Sicherheit - Wirtschaft - Öffentlichkeit}

Am Lehrstuhl für internationale Beziehungen der Universität zu Köln ist unter der Herausgeberschaft von Thomas Jäger, Alexander Höse und Kai Oppermann ein Sammelband entstanden, der sein Programm im Titel trägt: ,Transatlantische Beziehungen. Sicherheit Wirtschaft - Öffentlichkeit'. Das Autorenregister zählt über 40 Personen. Nahezu alle sind aus Deutschland oder den USA. Da es sich bei diesem Sammelband meist um Aufsätze handelt, die nicht eigens für dieses Vorhaben geschrieben wurden, sondern anderswo zwischen 2002 und 2004 erschienen sind, verwundert der übergroße Anteil deutscher und amerikanischer Autoren.

Die Herausgeber begründen ihre Auswahl ebenso wenig wie ihr Ziel und die Kriterien, die bei der Zusammenstellung der Beiträge angewandt wurden. Im Vorwort schreibt Jäger, dass die transatlantischen Beziehungen ,,seit jeher sämtliche Sachbereiche der Politik: Sicherheit, Wohlfahrt und Herrschaft" berührten. Deswegen könne ihre Analyse nicht auf ein einzelnes Politikfeld beschränkt werden, sondern müsse das Verhältnis in seiner Gesamtheit erfassen. Was hier mit Gesamtheit gemeint ist, benennen die drei Begriffe im Untertitel, denen sich die Hauptteile des Buches widmen. Der Bereich Sicherheit wird dabei noch einmal unterteilt in ein Kapitel, das sich mit Sicherheits- und Verteidigungspolitik befasst und ein weiteres, in dem es um Innere Sicherheit und Terrorismusbekämpfung geht.

Auf einen gemeinsamen analytischen Rahmen, eine theoretische Fundierung, eine übergreifende Fragestellung oder Zielsetzung wird verzichtet. Konsequenterweise wird im Schlusskapitel auch nicht versucht, eine Schlussfolgerung zu ziehen. Stattdessen wer- den unter dem Titel „Ausblicke“ fünf verschiedene Beiträge von namhaften Persönlichkeiten aus Politik und Wissenschaft (unter anderen Henry Kissinger, Lawrence Summers, Egon Bahr, Andrew Moravcsik) nebeneinander gestellt.

Inhaltlich bewegen sich die Einzelbeiträge zwischen drei Szenarien, die sich vom semantischen Aufwand her sehr, in ihrer Ausrichtung jedoch kaum unterscheiden: Europa steht an der Seite der USA. Es positioniert sich gegen die USA. Oder es findet einen dritten Weg dazwischen.

In vielen Beiträgen ist die Rede von starken Veränderungstendenzen und Dynamiken. So sieht Jäger in der Globalisierung (definiert als eine Komprimierung von Raum und Zeit), dem unterschiedlich verlaufenden Wertewandel auf beiden Seiten des Atlantiks und in der technischen Revolution der Kriegsführung seine drei zentralen Erklärungsfaktoren für die konstatierten Turbulenzen zwischen den USA und Europa (S. 19). Beiträge aus den anderen Bereichen kommen zu anderen Gewichtungen, Erklärungen und Bewertungen. Es lässt sich aber trotz der fachspezifischen Unterschiede ein gemeinsamer Nenner erkennen in Gestalt der weit verbreiteten Überzeugung, dass sich Veränderungen in den transatlantischen Beziehungen vor allem über eine unterschiedliche Entwicklung der Werte auf beiden Seiten des Atlantiks erklären lassen.

Da sich die Herausgeber konsequent einer Kommentierung und Interpretation der zusammengetragenen Beiträge enthalten, bleibt es dem Leser der 540 Seiten überlassen, das Verbindende oder einen roten Faden zu erkennen. Ohnehin ist aber kaum davon auszugehen, dass viele Leser ein solches Buch von vorn nach hinten durchlesen. Stattdessen mag wohl eher das Konzept verfolgt worden sein, ein Spektrum dessen anzubieten, was aktuell zu den als zentral erachteten Bereichen transatlantischer Beziehungen veröffentlicht wurde. So gesehen ist der Band ein durch seine Vielfalt interessantes Tableau, das vor 
allem dank seiner umfangreichen empirischen Übersichten, Tabellen und Synopsen auch als Nachschlagewerk nützlich erscheint. Wer sich rasch über den Stand von Entwicklungen bei Truppenstärken, Budgets oder der Gesetzgebung gegen Terrorbedrohung in komparativer Perspektive informieren will, findet hier kompetente und übersichtliche Antworten. Wer ein Gesamtbild sucht, steht angesichts der Fülle des Materials vor einer ziemlichen Herausforderung.

\section{Der Irakkrieg im transatlantischen Eliten- diskurs}

Eine andere Art Sammelband ist ,EU-US Relations. Repairing the Transatlantic Rift ${ }^{*}$, herausgegeben von Nikos Kotzias und Petros Liacouras. Der Titel ist auch in diesem Fall Programm. Für das Projekt wurden fast 40 Persönlichkeiten aus vielen verschiedenen Ländern und beruflichen Hintergründen versammelt, darunter George Soros, Gilles Andréani, Christoph Bertram, Kemal Dervis, Timothy Garton Ash, Stanley Hoffmann, Bronislaw Geremek oder Joseph Stiglitz. Angestoßen hatte das Projekt der damalige griechische Außenminister George A. Papandreou, der den Rahmen eines informellen EUAußenministertreffens in Kastellorizo während der griechischen Ratspräsidentschaft im Mai 2003 dazu nutzte, eine illustre Schar von europäischen und amerikanischen Spezialisten zu versammeln. Ihre gemeinsame Aufgabenstellung war es, unmittelbar nach Kriegsbeginn im Irak nach Antworten für das Problem zu suchen, wie die beschädigten transatlantischen Beziehungen repariert werden könnten. Vor diesem Hintergrund liest sich das Namensregister nicht nur wie ein Who is Who der transatlantischen Beziehungen. Neben der Qualität der Autoren liegt eine weitere Stärke des Bandes darin, dass die Beiträge auf die gemeinsame Aufgabenstellung verpflichtet wurden. Da das Buch aus einer Konferenz hervorging, hatten alle Autorinnen und Autoren Gelegenheit zum direkten Austausch und schrieben auf der Grundlage der gleichen historischen Situation mit dem Wissensstand vom Frühling 2003.

Die gemeinsame Arbeit entstand in unmittelbarer Nähe zur Politik, im nicht nur räumlichen Umfeld eines EU-Außenministertreffens. Es geht den Beiträgen folglich nicht allein um wissenschaftliche Hintergrundanalysen oder zeithistorische Bestandsaufnahmen, sondern vorrangig um politische Zuarbeit, Handlungsempfehlungen beziehungsweise konkrete Politikberatung.

Kotzias und Liacouras gliedern die Beiträge in sechs Kapitel. Nach einer Einführung der Herausgeber, die die Ausgangslage und die Aufgabenstellungen umreißt, folgen Teile, die sich in unterschiedlicher Perspektive mit den Ursachen der EU-US-Differenzen befassen. Daran schließen sich Politikempfehlungen an. Der Schlussteil enthält zwei längere Beiträge. Der erste stammt von Margarita Mathiopoulos, die den Entwurf einer neuen Transatlantischen Charta vorlegt. Dies erscheint - ohne dass das ausdrücklich als solche benannt wird - als eine Art Synthese des Buches. Daran schließt sich als Hintergrundmaterial eine Studie des Centre for European Studies in Birmingham an, die auch den Außenministern bei ihrem Treffen in Kastellorizo vorgestellt wurde. Darin geht es um die Untersuchung der Einstellungen der Europäer zu den transatlantischen Beziehungen für den Zeitraum 2000-2003.

Bietet der Kölner Sammelband einen Markt an Ideen und ein Spektrum an möglichen Entwicklungspfaden für die transatlantischen Beziehungen, so verfolgt das griechische Projekt das umgekehrte Ziel. Vor dem Hintergrund des gerade begonnenen Irakkriegs geht es nicht darum, Alternativen auszuloten, sondern um ein eindeutiges politisches Bekenntnis zu Europa als verlässlichem, konstruktiven Partner der USA.

Jenseits seiner politischen Funktion und der analytischen Qualität der einzelnen Beiträge ist der Band auch zeitgeschichtlich interes- 
sant. Die Momentaufnahme vom Frühling 2003, wo eine transatlantische Elite kurz nach dem Beginn des Irakkriegs über die Möglichkeiten zur Reparatur der Beziehungen nachdenkt, liefert nicht nur ein Tableau der dort vertretenen Meinungen. Sie ist wegen der Nähe der Beteiligten zur europäischen Politik auch eine Quelle; beispielsweise für die Antwort auf die Frage, welche Werte und Einstellungen handlungsleitend gewesen waren an diesem Punkt in der Geschichte der transatlantischen Beziehungen.

\section{USA und EU - Revolutionary Powers?}

An der Universität Edinburgh entstand unter der Herausgeberschaft von Roland Dannreuther und John Peterson ein Sammelband, der sich auf Aspekte der Sicherheitspolitik in den transatlantischen Beziehungen konzentriert. Im Mittelpunkt von ,Security Strategy and Transatlantic Relations" steht der Vergleich der Europäischen mit der Amerikanischen Sicherheitsstrategie. Der Band ist mit 200 Textseiten weniger als halb so umfangreich wie die zuvor besprochenen Bücher. Lag deren Stärke in der Breite und Vielfalt, so besticht das schottische Projekt durch seinen Tiefgang. Es hat einen thematischen Fokus, eine gemeinsame Fragestellung und es wird im Einführungsteil eine historische Einbettung und eine theoretische Fundierung geliefert, von der aus die einzelnen Beiträge weiterarbeiten. Die Herausgeber führen ein kleines, überwiegend schottisches Team an Wissenschaftlern konzeptionell zu einem imposanten Gemeinschaftswerk, was mehr ist als die Summe der einzelnen Teile und was seit seinem Erscheinen in der Fachwelt breit und ausgesprochen positiv aufgenommen wurde.

Wie auch in den anderen besprochenen Büchern geht es auch hier den Autoren jenseits der empirischen Analysen im Kern um die Frage nach den Grundlagen der Beziehungen zwischen den USA und Europa. Verschiedene Beiträge spezifischer Politikfeldanalysen betonten, dass der Kontext oder exogene Fakto- ren das transatlantische Verhältnis veränderten. Der Befund wurde mitunter verbunden mit der Hoffnung, dies möge nichts an den gemeinsamen Grundwerten und Überzeugungen ändern. Bei Dannreuther/Peterson werden die Beziehungen zwischen den USA und Europa nicht nur als abhängige Variable diskutiert, sondern explizit auch als unabhängige Variable und hier unter dem Aspekt von USA und EU als ,revolutionary powers“.

In dieser Lesart werden die Sicherheitsstrategien als Versuche der USA und der EU verstanden, die Welt nach jeweils ihrem Abbild zu gestalten. Dies wirft in den einzelnen Beiträgen eine Vielzahl von Fragen auf, wie sie sich zu Zeiten des Kalten Krieges so nicht gestellt haben. Eine solche Perspektive unterscheidet sich aber nicht nur methodisch von den meisten anderen Beiträgen in den besprochenen Büchern. Sie weist zugleich über die reine Binnenanalyse hinaus und fragt nicht nur nach den Folgen der Veränderungen für die USA und Europa, sondern für die Welt.

Ob aber diese revolutionäre Umgestaltung gemeinsam, gegeneinander oder in einer irgendwie gearteten Zwischenform geschehen wird, hängt wieder von den Grundlagen ab. Diese auszuloten geht über die Möglichkeiten eines solchen Sammelbandes hinaus, auch wenn er klug konzipiert ist und auf kompetente Einzelbeiträge bauen kann.

\section{,Atlantische Zivilisation“}

Lazaros Miliopoulos legt mit seiner Dissertation einen Beitrag zur Grundlagenforschung vor. Motiviert von der verlorenen Gewissheit darüber, was den ,Westen ' ausmacht, liefert seine Doktorarbeit mit dem Titel ,Atlantische Zivilisation und transatlantisches Verhältnis. Politische Ideen und Wirklichkeit" nicht nur eine höchst belesene und intellektuell anspruchsvolle ideengeschichtliche und geschichtswissenschaftliche Bestandsaufnahme der ideellen Grundlagen der transatlantischen Beziehungen. Die Arbeit verfolgt auch ein politisches Ziel, nämlich ,ein Zivilisationsmo- 
dell als politische Idee des Westens systematisch vor dem Hintergrund einer historischen Wirklichkeit zu entwerfen, sowie diese Idee als alternatives Angebot in die zivilisationspolitische Debatte einzubringen“ (S. 41). Nach seinem Befund leiden die Beziehungen zwischen Europa und den USA an ,einer Identitätskrise aufgrund eines fehlenden Identitätsbewusstseins“ (S. 41). Das gilt es ihm wiederherzustellen.

Vor diesem Hintergrund will Miliopoulos nichts weniger als eine philosophische Idee des Westens entwickeln, für die er den Begriff der „Atlantischen Zivilisation“ wählt und die mit dem Anspruch konkreter Realisierbarkeit in die politische Praxis eingebracht werden soll. Sein Sprachniveau und ein über 2.500 Fußnoten umfassender Apparat erleichtern die Anbindung an die zivilisationspolitische Debatte nicht gerade, aber das ist auch nicht die vorrangige Aufgabe einer
Dissertation auf dem Feld der Grundlagenforschung.

Was dabei verarbeitet und mit Blick auf das selbst gesteckte Ziel geleistet wird, verdient hohe Anerkennung. Ob und in welcher Weise diese politische Idee den Diskurs befruchtet, ist kein Maßstab für ihre Qualität, liegt das doch jenseits der Möglichkeiten einer solchen Arbeit.

Angesichts der Heterogenität der besprochenen Veröffentlichungen verbietet sich ein Fazit, weil es den unterschiedlichen Ansprüchen und Qualitäten jeder einzelnen nicht gerecht werden würde. Als eine Art roter Faden zieht sich aber ein Eindruck durch alle Bücher, egal ob als politisches Programm explizit formuliert oder als Unterton: Die ideellen Grundlagen des Verhältnisses zwischen den USA und Europas zu suchen, bewusst zu machen und in die politische Praxis zu übersetzen, ist aller Mühen wert.

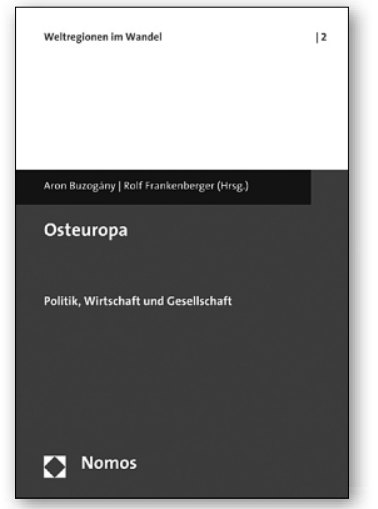

\section{Osteuropa}

Politik, Wirtschaft und Gesellschaft

Herausgegeben von Aron Buzogány und

Rolf Frankenberger

2007, 292 S., brosch., 45,-€, ISBN 978-3-8329-3064-6

(Weltregionen im Wandel, Bd. 2)

Ausgehend von Grundüberlegungen zur Entwicklung der Transformationsforschung sowie der Bedeutung von Zivilgesellschaft in Demokratisierungsprozessen erörtert der vorliegende Band exemplarisch zentrale Entwicklungsdynamiken, welche den Wandel von Staat, Wirtschaft und Gesellschaft in Osteuropa in den letzten beiden Dekaden prägen.

Bitte bestellen Sie bei Ihrer Buchhandlung oder bei Nomos | Telefon 07221/2104-37 | Fax -43 www.nomos.de | sabine.horn@nomos.de

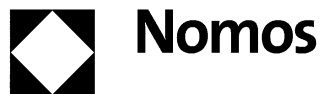

